\title{
Characteristics of Multidrug Resistant Tuberculosis in Minia, Egypt
}

\author{
Shimaa Anwer Emam ${ }^{1}$ Eman Mahfouz Kasem², Amany Edward Sedhom² \\ ${ }^{1}$ Lecturer, ${ }^{2}$ Professor, of Public Health and Preventive Medicine Department, Faculty of Medicine, Minia \\ University, Egypt
}

\section{Background}

Tuberculosis (TB) is a disease of great antiquity and has almost certainly caused more suffering and death than any other infection. A total of 40 patients were registered with a diagnosis of MDR-TB during 20102018 in Minia. The majority of patients were from rural areas $(27 ; 67.5 \%)$ and male $(27 ; 67.5 \%)$. Emergence of MDR-TB has the potential to be a serious public health problem in that necessitates strengthened TB control and improved continuous monitoring of therapy.

Keywords: TB; $M D R-T B$; Therapy

\section{Introduction}

TB is a major public health problem owing to its high risk of person-to-person transmission, morbidity, and mortality. TB still remains one of the major health miseries facing humans, particularly in developing countries (1).

Multidrug-resistant TB (MDR-TB) remains a public health crisis and a health security threat. World Health Organization estimates that there have been 558000 new cases with resistance to rifampicin - the foremost effective first-line drug, of that $-82 \%$ had MDR-TB.

According to the latest WHO estimation the incidence of tuberculosis (per 100,000 people) in Egypt was reported at 13 in 2017 (2). Screening, diagnosis, notification, and registration of TB cases were implemented all over Egypt according to the National TB Strategy of the National Tuberculosis Control Program (NTP). One of the registration sites is Minia

\footnotetext{
Corresponding author

Shimaa Anwer M.D.

Lecturer of Public Health and Preventive Medicine Department of Public Health, Faculty of Medicine, ElMinia University, University St., El-Minia 1666, Egypt Tel: +2 -086-2367252, Mobil: 01001934971

E-mail address@yahoo.com”shimaa_anwer3@yahoo. com
}

Chest Hospital, Minia, Egypt where the current study was conducted.

\section{Method}

This was a hospital-based retrospective study conducted to detect the pattern of prevalence, risk factors and treatment outcomes among patients with multidrugresistant tuberculosis (MDR-TB) in Minia, Egypt and involving a record review of patients with TB notified and registered in Minia Chest Hospital. Data of 40 MDRTB cases reported from January 1, 2010 to December 31, 2018 were analyzed. The diagnosis of MDR-TB in Minia Chest Hospital is made in line with the National Egyptian TB Control Program Guidelines of the Ministry of Health (NTP). Sociodemographic characteristics, associated comorbidities, fate of outcome and regimens of previous antituberculous treatments received either category I (CAT1) (Isoniazid "H", rifampicin "R", pyrazinamide "Z", ethambutol "E" with or without streptomycin "S" for 2 months followed by isoniazid and rifampicin for 4 months $\{2 \mathrm{HRZE}(\mathrm{S}) / 4 \mathrm{HR}\}$ ), category II (CAT2) (2HRZES/1HRZE/5HRE) were collected. Cured patient was defined as a patient who is smear-negative in the last month of treatment and on at least one previous occasion. Treatment completed was defined as a patient who has completed treatment but who does not meet the criteria to be classified as a cure or a failure. Death was defined as a patient who dies for any reason during the course of treatment. Treatment default was defined as a patient whose treatment was interrupted for two months 
or more. Treatment failure was defined as a patient who remained or became again smear-positive at five months or later during treatment or defined as a patient who was initially smear-negative before starting treatment and become smear-positive after completing the initial phase of treatment. Trend curve of prevalence rate of MDR-TB cases in Minia, Egypt from 2003 to 2018 was performed.

\section{Limitation of the study}

By using retrospective data there were unavailability of clinical data, radiologic and other laboratory investigations.

\section{Statistical Analysis}

Data analysis was performed using SPSS version 20 (IBM Corp. Released 2011. IBM SPSS Statistics for Windows, Version 20.0. Armonk, NY: IBM Corp. US). Crude and adjusted odds ratios (ORs) and their 95\% confidence intervals (CI95) were calculated. A binary logistic multivariate model was applied to determine the independent predictors of the treatment outcome out of those factors that demonstrated significant association by bivariate analysis at a level of significance of $p \leq 0.05$.

\section{Results}

A total of 40 patients were registered with a diagnosis of MDR-TB during 2010-2018 in Minia. The majority of patients were from rural areas $(27 ; 67.5 \%)$ and male $(27 ; 67.5 \%)$. The mean age of the patients was 38 years
( $\mathrm{SD} \pm 14.3$ years). Smokers represented $55 \%$ of patients. Married patients were $60 \%$ of patients (Table1).

Majority of patients received CAT1 treatment at home and about half of them were regular in treatment. The commonest side effects of drugs used in treatment of MDR-TB patients were GIT symptoms and peripheral neuritis as shown in (Table 1).

The adjusted odds ratios (OR) and $95 \%$ confidence intervals (CI) for the association between the combined effect of independent variables and the outcome variable (MDR-TB). These estimates were obtained by logistic regression analysis. Marital status, place of previous treatment, compliance and associated comorbidities were statistically associated with. Compliance to treatment was found to be the most important determinant (Table 2).

Regarding the associated co-morbidities, $2.5 \%$ of the included patients were positive for HIV, $17.5 \%$ of them had cardiac diseases, $25 \%$ were diabetics, $10 \%$ had $\mathrm{HCV}$ and 10\% had chronic chest diseases (Fig. 1).

Patients completed treatment performed $37.5 \%$ and cases with favorable outcome were $22.5 \%$ while failure of treatment were only $5 \%$ (Fig. 2).

The trend curves of MDR-TB and TB cases in Minia were decreasing in the years 2004, 2010, 2012 and 2018. The trend curves were increasing in the years 2005and 2011 (Fig 3).

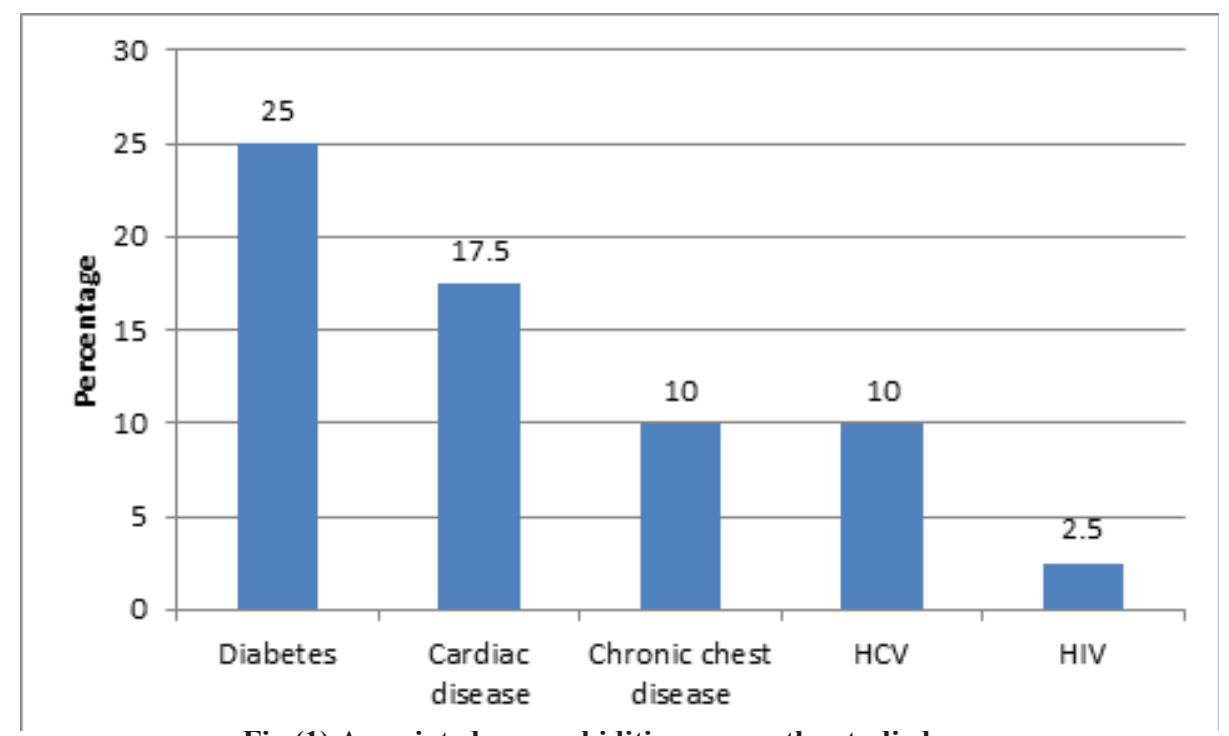

Fig (1) Associated co morbidities among the studied cases 


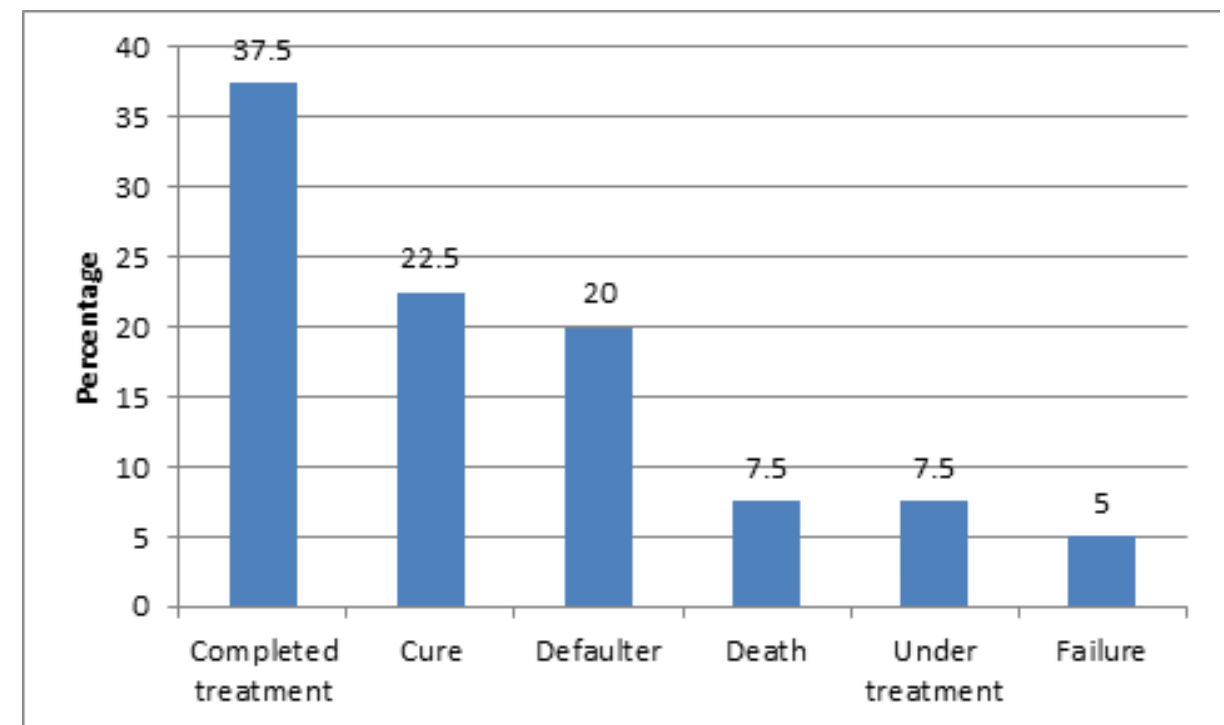

Fig (2): Outcome of TB treatment among the studied cases

Fig (3): Trend in prevalence of MDR- TB among TB patients in the period from 2003 to 2018

Table (1): Characteristics of MDR-TB cases in the period 2010-2018, Minia, Egypt

\begin{tabular}{|c|c|c|c|}
\hline Age (years) & Mean \pm SD & \multicolumn{2}{|c|}{$38.6 \pm 14.3$} \\
\hline & & No. & $\%$ \\
\hline \multirow{2}{*}{ Sex } & Male & 27 & 67.5 \\
\hline & Female & 13 & 32.5 \\
\hline \multirow{2}{*}{ Smoking status } & Smoker & 22 & 55 \\
\hline & Non smoker & 18 & 45 \\
\hline \multirow{2}{*}{ Residence } & Rural & 27 & 67.5 \\
\hline & Urban & 13 & 32.5 \\
\hline \multirow{4}{*}{ Occupation } & Non-worker & 13 & 32.5 \\
\hline & Manual & 6 & 15 \\
\hline & Farmer & 13 & 32.5 \\
\hline & Housewife & 8 & 20 \\
\hline \multirow{4}{*}{ Marital status } & Single & 5 & 12.5 \\
\hline & Married & 24 & 60 \\
\hline & Widow & 7 & 17.5 \\
\hline & Divorced & 4 & 10 \\
\hline \multirow{2}{*}{ Place of treatment } & Home & 36 & 90 \\
\hline & Hospital & 4 & 10 \\
\hline \multirow{2}{*}{ Regimen of treatment } & CAT1 & 37 & 92.5 \\
\hline & CAT2 & 3 & 7.5 \\
\hline
\end{tabular}


Cont... Table (1): Characteristics of MDR-TB cases in the period 2010-2018, Minia, Egypt

\begin{tabular}{|c|l|l|l|}
\hline \multirow{4}{*}{ Compliance } & Regular & 21 & 52.5 \\
\cline { 2 - 4 } & Irregular & 7 & 17.5 \\
\cline { 2 - 4 } & Uncertain & 12 & 30 \\
\hline \multirow{4}{*}{ Side effects } & Nothing & 16 & 40 \\
\cline { 2 - 5 } & GIT symptoms & 18 & 45 \\
\cline { 2 - 4 } & Peripheral neuritis & 2 & 5 \\
\cline { 2 - 4 } & Arthritis & 4 & 10 \\
\hline Total & & 40 & \multicolumn{2}{|c|}{100} \\
\hline
\end{tabular}

Table (2): Logistic regression analysis of factors affecting MDR-TB among the studied cases, Minia, Egypt

\begin{tabular}{|l|l|l|l|}
\hline Variables & OR & CI & P-value \\
\hline Age & 0.95 & $0.86-1.04$ & 0.26 \\
\hline Sex & 1.1 & $0.075-16.5$ & 0.9 \\
\hline Occupation & 1.9 & $0.7-5.04$ & 0.18 \\
\hline Residence & 2.006 & $0.28-14.6$ & 0.5 \\
\hline Marital status & 5.6 & $1.1-28.7$ & $0.03^{*}$ \\
\hline Smoking & 4.5 & $0.4-51.5$ & 0.2 \\
\hline Place of previous treatment & 4.6 & $3.08-26.2$ & $0.04 *$ \\
\hline Regimen of previous treatment & 3.02 & $0.006-1509.8$ & 0.7 \\
\hline Compliance to treatment & 2.03 & $1.3-3.9$ & $0.02^{*}$ \\
\hline Side effects of drugs & 1.3 & $0.65-2.5$ & 0.84 \\
\hline Associated comorbidities & 1.95 & $1.57-2.6$ & $0.04 *$ \\
\hline
\end{tabular}

\section{Discussion}

Multi Drug Resistance Tuberculosis MDR-TB is a rapidly increasing public health problem with major socio-economic and individual consequences. The spread of MDR-TB can only be prevented by rapid identification of these cases and treatment with a combination of effective drugs ${ }^{(5)}$.

In the present study, males constituted $67.5 \%$ while females represented $32.5 \%$. This coincides with the epidemiological picture of tuberculosis where males spend more number of hours outdoor exposure and more challenging and hazardous working environments. Also this came in accordance with ${ }^{(6)}$ who reported that the percentage of MDR-TB among males was $75.9 \%$ and that of females was $24.1 \%$ in three different governorates in Egypt.

In this study, we found that $55 \%$ of MDR-TB patients were smokers and this coincided with that of (7) in El-Abbasia Chest Hospital who revealed that in MDR TB, smokers were $61.53 \%$ and non-smokers were $38.47 \%$ among MDR-TB cases.

In many countries, differences in MDR-TB prevalence rates between urban and rural areas have been described. In the current study, $62.5 \%$ of patients were from rural areas and $32.5 \%$ were from urban areas. A study by ${ }^{(8)}$ has reported similar findings that $90 \%$ were 
from rural areas and only $10 \%$ were from urban area in the Dakahlia governorate from 2006 to 2011. Increased MDR-TB cases in rural areas could be explained by poverty, bad social conditions, milk sanitation, and occupational exposure to infected animals.

In this study, the most common co-morbidity associated with MDR TB was diabetes. This result agreed with those of ${ }^{(9)}$ who reported that the highest co-morbidity among MDR-TB patients was DM 29.9\%. Also, it was matched with those of ${ }^{(10)}$, who reported that the highest co-morbidity among MDR-TB patients was DM (18.3\%) of the patients.

In the present study, cured patients were $(22.5 \%)$, dead patients were $(7.5 \%)$ and defaulters were $(20 \%)$. This result was matched with those of ${ }^{(11)}$, who reported that (19.4\%) were successfully treated, $(20.9 \%)$ died, defaulted (13.4\%). On the other hand, the result didn't agree with ${ }^{(12)}$, who studied the outcome of treatment of MDR TB patients in Russia that $76.0 \%$ was cured. Again, the result didn't match with those of ${ }^{(13)}$ who reported that $70.6 \%$ were cured in United Kingdom from 20042007. This difference might be due to patient compliance with treatment and regular drug in developed countries.

In the present study, $45 \%$ of patients suffered from gastrointestinal disorders. This result coincided with those of (14), who stated that the most frequent side effect of Anti TB drugs was gastrointestinal manifestations (64\%). Also, this result agreed with those of (10), who reported that dominant adverse effect was gastrointestinal disorders (55\%). Oppositely, the result did not coincide with those of (15) who reported that the highest adverse effects were ototoxicity $41.8 \%$, psychological $21.3 \%$ and gastrointestinal $14 \%$. This difference might be due to the fact that Törün study included 263 MDR TB patients who received individualized treatment for MDR-TB between April 1992 and June 2004 at Istanbul, Turkey and also the author said that the frequent and early occurrence of ototoxicity may be due to the extended exposure to amino glycosides and Capreomycin during or prior to MDR-TB treatment.

In the current study, the studied cases were resistant to CAT1 (rifampicin, isoniazid, ethambutol and streptomycin) represented $92.5 \%$. This result agreed with the findings reported by (16) that $65 \%$ of patients were resistant to CAT1. Contrary to our results (17) in Bulgaria founded that only $52 \%$ of cases were resistant to CAT1 this high percent of acquired resistance may be due to alcoholism, number of previous treatments and irregular treatments. A study by (18) reported that only $42.8 \%$ of patients in a tertiary hospital in India were resistant to CAT1.Such variation may be due to varied geographical distribution, circulating strain patterns, demographic, ethnic, and epidemiological differences.

In this study comorbidities increased risk of MDRTB $(p=0.04)$ and this was observed by (19) in South Korea who found that relapse with resistant strains and poor treatment outcome of MDR-TB was documented among comorbid patients. Active screening for diabetes and HIV among TB patients is suggested as a costeffective measure to be incorporated within the TB control program.

Our study revealed that MDR-TB infection had a statistically significant association with patients place of previous treatment $(\mathrm{p}=0.04)$ and this is in agreement with study done in Addis Ababa, Ethiopia by (20) on patients who visited health facilities $(\mathrm{p}<0.005)$. Compliance to treatment appeared to be a predictor for MDR-TB $(\mathrm{p}=0.02)$ and this finding was in agreement with a study by $(21)$ in China $(p<0.005)$, which requires strong commitment and collaboration among health organizations and greater compliance with TB treatment guidelines by service providers and patients.

In the present study, the trend curve of MDRTB cases in Minia was decreasing in the years 2004, 2010, 2012 and this may be due to that the program of MDR-TB was approved and implemented in Egypt in the year 2003, by the Green Light Committee (GLC) (22). The trend curve was increasing in the years 2005 and 2011with more detection rate of drug resistant TB cases with drug susceptibility tests and increasing slum areas, low socioeconomic status and poor nutrition and political disturbance and 25th January revolution.

\section{Conclusion}

Emergence of MDR-TB has the potential to be a serious public health problem in that necessitates strengthened TB control and improved continuous monitoring of therapy. Emphasis on the need to complete medical records of patients in hospitals.

\section{Declarations:}

- There is no conflicts of interest and/or funding

- The paper has been read and approved by all authors. 
- Ethical considerations: The study was approved by the ethical committee of the Faculty of Medicine, Minia University. Prior to data collection, official permissions were obtained from the authorities of Minia University Hospital. Following the ethical guidelines of epidemiological research, a written informed consent was taken from each participant.

- Data is available on request.

Ethical approval: Ethics approval to use, report, and publish the collected data was obtained from the administrator of Minia Chest Hospital. Patient information was anonymized and deidentified prior to the analysis. Research Ethics Committe at the Faculty of Medicine, Minia University, approved this study protocol.

\section{Refrences}

1- Pande JN. Tuberculosis research in India. Indian Journal of Medical Research 2004; 120:205-206.

2- World Health Organization https://www.who.int/tb/ country/data/profiles/en 25/9/2018. Accessed on 11/12/2018.

3- Pablos-Mendez A, Raviglione M.C. , Laszlo A, Binkin N, Rieder H.L., Bustreo F. Global surveillance for antituberculosis-drug resistance, 1994-1997. World Health OrganizationInternational Union against Tuberculosis and Lung Disease Working Group on Anti-Tuberculosis Drug Resistance Surveillance, N. Engl. J. Med. 338 (23) (1998) 1641-1649.

4- World Health Organization Guidelines for the programmatic management of drug-resistant tuberculosis. WHO/HTM/2006.361 The World Health Organization, Geneva (2006).

5- Weiss P, Chen W, Cook J.V, Johnston J .Treatment outcomes from community-based drug resistant tuberculosis treatment programs: a systematic review and meta-analysis BMC Infect Dis, 14 (2014), p. 333.

6- Emad Ibrahim, Mahmoud Aly Al Messery, Pattern of prevalence, risk factors and treatment outcomes among Egyptian patients with multidrug resistant tuberculosis. Egyptian Journal of Chest Diseases and Tuberculosis. 2017; 66:3: 405-411.

7- Kamal, A. Khattab, M. Mansour, Multiple Drugresistant Tuberculosis at Abbassia Chest Hospital from January 2006 to December 2005, Thesis submitted for partial fulfillment of Master Degree in Chest Diseases and Tuberculosis, Ain Shams University, 2007.

8- Medhat F Negm, Amira H Allam, Fatehia S El Zeheiry. Assessment of directly observed therapy short-course (DOTs) of tuberculosis in Dakahlia governorate chest hospitals from 2006 to 2011. The Egyptian Journal of Bronchology. 2017: 11 (2): 8897.

9- Mohammad A.Tag El Din, Ashraf A.El Maraghy, Abdel Hay R.Abdel. Adverse reactions among patients being treated for multi-drug resistant tuberculosis at Abbassia Chest Hospital. Egyptian Journal of Chest Diseases and Tuberculosis . 2015; 64: 4: 939-952.

10- Safwat TM, ElMasry AA, Mohamed M. Prev'alence of multi drug-resistant tuberculosis at Abbassia Chest Hospital from July 2006 to December 2009. Egyptian J. Bronchol., 2011; 5:2.

11- Isaakidis P, Varghese B, Mansoor H, Cox H S, Ladomirska. Adverse events among HIV/MDRTB co-infected patients receiving antiretroviral and second line anti-TB treatment in Mumbai, India PLoS ONE. 2012; 7 (7): e40781.

12- Shin SS, Pasechnikov A.D., Gelmanova I.Y., Peremitin G.G., Strelis A.K., Mishustin S, Barnashov A, Karpeichik Y, Andreev V.T. Golubchikova T.P, Tonkel G.V, Yanova A,Yedilbayev M.L, Rich J.S, Mukherjee J.J. Adverse reactions among patients being treated for MDR-TB in Tomsk, Russia Int. J. Tuberc. Lung Dis. 2007; 11:12: 1314-1320.

13- AndersonS., Tamne JP, WatsonT., Cohen C.D., Mitnick T. Treatment outcome of multidrug resistant tuberculosis in the United Kingdom: retrospective-prospective cohort study from 2004 to 2007 Euro Surveillance. 2013; 18 (40): 20-26.

14- El-Naggar T.A.H, Dewidar M., Nada A.F. Evaluation of Outcome of Multi-Drug Resistant Antituberculous Treatment at Abbassia Chest Hospital between July 2006 to June 2008. (2009) 135-136.

15- Törün T, Güngör Y, Özmen, I. Bölükba, E. Maden, B. Bıçakçı, G. Ataç, T. Sevim, K. Tahaolu. Side effects associated with the treatment of multidrugresistant tuberculosis. Int. J. Tuberc. Lung Dis. 2005; 9: 12: 1373-1377.

16- Daniel O, Osman E. Prevalence and risk factors associated with drug resistant $\mathrm{TB}$ in South 
West, Nigeria. Asian Pacific Journal of Tropical Medicine 2011, pp148-151.

17- Dhingra V K, Rajpal S, Mittal A, Hanif M. Outcome of multi-drug resistant tuberculosis cases treated by individualized regimens at a tertiary clinic. Indian Journal of Tuberculosis, (2008); 55, pp. 15-21.

18- Jain A, Diwakar P, Singh U. Declining trend of resistance to first line anti-tubercular drugs in clinical isolates of Mycobacterium tuberculosis in a tertiary care north Indian hospital after implementation of revised national tuberculosis control program. Indian J Med Microbiol. 2014; 32:430- 433.

19- Kim DH, Kim HJ, Park SK, Kong SJ, Kim YS et al. Treatment outcomes and long-term survival in patients with extensively drug-resistant tuberculosis. Am J Respir Crit Care Med, (2008)
178: $1075-1082$.

20- Abate D, Taye B, Abseno M, Biadgilign S. Epidemiology of anti-tuberculosis drug resistance patterns and trends in tuberculosis referral hospital in Addis Ababa, Ethiopia. BMC Res Notes. 2012; 5:462.

21- Liang L, Wu Q, Gao L, Hao Y, Liu C, Xie Y, et al. Factors contributing to the high prevalence of multidrug-resistant tuberculosis: a study from China. Thorax BMJ. 2012; 67(7): 632-638.

22- Kai Blöndal. Barriers to reaching the targets for tuberculosis control: multidrug-resistant tuberculosis. Bull World Health Organ. 2007 May; 85(5): 387-390. 